\title{
Texture Feature Extraction based on Local Weighting Pattern (LWP) using Fuzzy Logic Approach
}

\author{
Achmad Fanany Onnilita Gaffar \\ Department of Information Technology \\ State Polytechnic of Samarinda \\ Samarinda, East Kalimantan - Indonesia
}

\author{
Agusma Wajiansyah \\ Department of Information Technology \\ State Polytechnic of Samarinda \\ Samarinda, East Kalimantan - Indonesia
}

\author{
Arief Bramanto Wicaksono Putra \\ Department of Information Technology \\ State Polytechnic of Samarinda \\ Samarinda, East Kalimantan - Indonesia
}

\begin{abstract}
The texture feature description becomes a tremendous challenge in the field of computer vision and pattern recognition. The high-quality feature descriptor attributes some of which are unique, due to a large number of texture classes, robust against illumination variations, and low dimensional representations. A number of image feature extraction methods had been proposed, which can be divided into two categories: holistic and local image feature extraction. The holistic feature extraction method is very sensitive to changes in geometric shapes and some variations of illumination and noise. The local image feature extraction methods can effectively overcome those weaknesses. In this study, the texture features of an RGB image are built using the Local Weighting Pattern (LWP). By using the gray-level dynamic range modification technique, Fuzzy Membership Function (MF) is applied to LWP texture images to build Fuzzy-based LWP image (FLWP). From the resulting image is then used to generate a feature descriptor in the form of labels.
\end{abstract}

\section{General Terms}

Image Processing, Fuzzy Logic

\section{Keywords}

Texture feature extraction, Feature descriptor, Local Weighting Pattern (LWP), Fuzzy Membership Function

\section{INTRODUCTION}

The texture is a description of the spatial arrangement of color or intensity in a selected image or image area. Textured areas in the image can be marked with uniform or varying spatial intensity distribution. Its intensity variations reflect some changes in the imaged scenes. There are several texture properties such as granularity, directionality, randomness or regularity and texture elements [1].

The texture feature description becomes a tremendous challenge in the field of computer vision and pattern recognition. The extraction of powerful texture features plays an important role. If a bad feature is used even the best classifier will fail to achieve a good recognition result. Most of the research in texture classification focuses on how to develop powerful feature extraction methods. The highquality feature descriptor attributes some of which are unique, due to a large number of texture classes, robust against illumination variations, and low dimensional representations. Many research efforts have been made to achieve either strict quality requirements or low computational speed [2].

A number of image feature extraction methods had been proposed, which can be divided into two categories: holistic and local image feature extraction. Holistic feature extraction method is the method based on a statistical information template from a large amount of training sample images. One of the typical methods is Principal Component Analysis (PCA). Other methods using decomposition technique and linear combination have become very popular, such as Linear Discriminant Analysis (LDA), Discrete Wavelet Transform (DWT), Kernel Support Vector Machine (KSVM), Gray Level Co-occurrence Matrices (GLCM), Gabor filters (GF), wavelet methods[3-10], etc. However, the holistic feature extraction method is very sensitive to changes in geometric shapes and some variations of illumination and noise. The local image feature extraction methods can effectively overcome those weaknesses. Some of these methods are local binary pattern (LBP) [11], Median Robust Extended LBP (MRELBP) [2], Local Derivative Pattern (LDP) [12], Local Tri-directional Weber Patterns (LTrWP) and Weber local descriptor (WLD) [13], Generalized Gabor direction pattern (GGDP) , Patch-structure Direction pattern (PDP), Weighted Discrepancy Measurement Model (WDMM) [9], edged image based [4], feature extraction method of Gabor Filter in rank fusion level and minutiae feature extraction method for feature level fusion [14], etc. Another method of feature extraction is an extraction process based on machine learning method [15]. The extraction of the feature of an image is used for various purposes, such as face recognition, object detection, fingerprint verification, content-based image retrieval [5], [8], [10], [12], [16], [17], [18], etc.

In this study, the texture features of an RGB image are built using the Local Weighting Pattern (LWP). By using the graylevel dynamic range modification technique, Fuzzy Membership Function (MF) is applied to LWP texture images to build Fuzzy-based LWP image (FLWP). From the resulting image is then used to generate a feature descriptor in the form of labels. 


\section{LOCAL WEIGHTING PATTERN (LWP)}

Pixel neighbors are a set of pixels where their location is relative to the center of the pixel. The neighborhood is a rectangular block, typically having $8,16,32$ pixels around the center of the pixel. The sliding neighbor operation is an operations performed on the center of pixel based on all neighboring pixels with a change in the center of pixel value determined by the application of a particular algorithm to the corresponding neighboring pixel input value. In this study uses 8-pixels neighbors. LWP is a pattern built based on the local weight of a pixel which is the sum of its binary code constructed based on all threshold values between neighboring pixels to the center of the pixel. There are two types of LWP codes, which are positive and negative, expressed as:

Positive code:

$$
\text { binarycode }(i)=\left\{\begin{array}{cc}
1 & \text { if } P\left(x_{c}, y_{c}\right)-P\left(x_{i}, y_{i}\right) \geq 0 \\
0 & \text { otherwise }
\end{array}\right.
$$

Negative code:

$$
\text { binarycode }(i)=\left\{\begin{array}{cc}
1 & \text { if } P\left(x_{i}, y_{i}\right)-P\left(x_{c}, y_{c}\right) \geq 0 \\
0 & \text { otherwise }
\end{array}\right.
$$

where, $P\left(x_{c}, y_{c}\right), P\left(x_{i}, y_{i}\right)$, and $i$ are a center of the pixel, its neighbors, and sequence number of binary code, respectively. This process as shown in Fig. 1.

sample
\begin{tabular}{|c|c|c|}
\hline 5 & 10 & 7 \\
\hline 20 & 13 & 30 \\
\hline 9 & 13 & 15 \\
\hline
\end{tabular}

difference
\begin{tabular}{|c|c|c|}
\hline-8 & -3 & -6 \\
\hline 7 & & 17 \\
\hline-4 & 0 & 2 \\
\hline
\end{tabular}

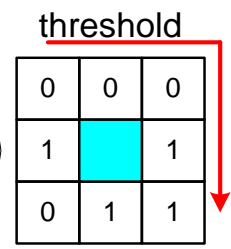

(a)

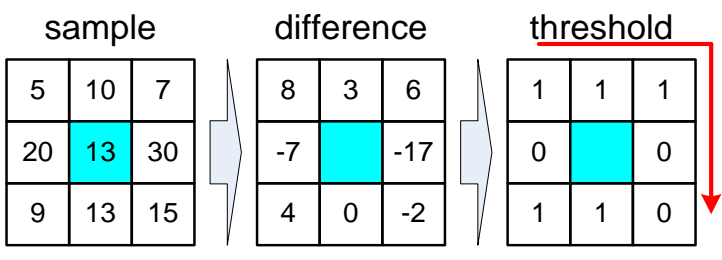

(b)

Fig. 1 Calculating the LWP code: (a). positive code, (b). negative code

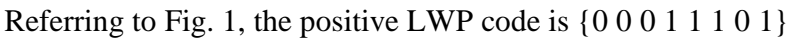
and the negative LWP code is $\left\{\begin{array}{llllllll}1 & 1 & 1 & 0 & 0 & 1 & 1 & 0\end{array}\right\}$. In this study uses positive LWP code. The LWP operator is the sum of all binary numbers of LWP codes declared by

weight $=\sum_{i=1}^{8}$ binarycode $(i)$

$P\left(x_{c}, y_{c}\right)_{\text {new }}=P\left(x_{c}, y_{c}\right)_{\text {new }} *$ weight

The image row and columns need to be expanded so that block processing can be performed on each pixel that is on the edge of the image, as shown in Fig. 2 (a). After the LWP operation is applied, each pixel value is normalized with its maximum pixel value to obtain an 8-bit image using the formula:
$P(x, y)_{\text {new }(i)}=\frac{P(x, y)_{\text {old }(i)}}{\max \left(P(x, y)_{\text {old }}\right)} \times 255$

The operation is shown in Fig. 2 (b).

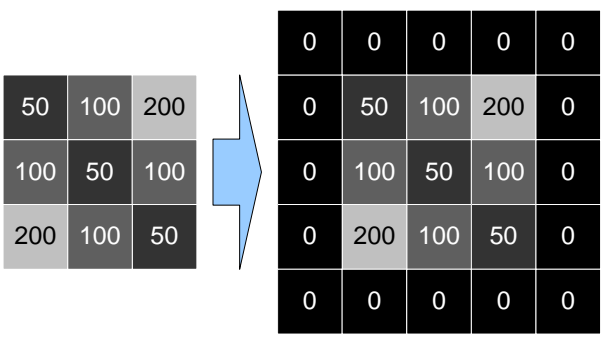

Sample Image Expanded sample Image

(a)

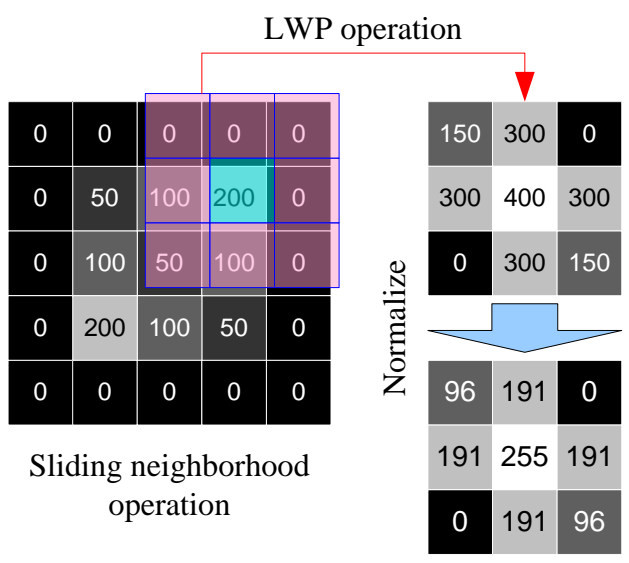

LWP texture Image

(b)

Fig. 2 (a) Image expansion, (b) Block processing which performed at each pixel

\section{FUZZY-BASED IMAGE}

The fuzzy-based image is built using a gray-level dynamic range modification technique which mapping the range of gray-level of the input image to the new range of gray-level as the output image. In principle this technique maps the gray level of the input image to a certain gray level expressed by

$g(x, y)=f(I(x, y))$

Where, $f(\quad)$ is the function that maps the gray level image $I(x, y)$ to $g(x, y)$. If $f()$ is a Fuzzy MF (Membership Function) it will generate an image $g(x, y)$ based on the various Fuzzy MF used.

Fuzzy MF implemented in the image will act as a filter that passes a certain pixel intensity value to a new pixel intensity value depending on the selection of its fuzzy MF type and parameter settings used. Some fuzzy MFs are shown in Fig. 3. 


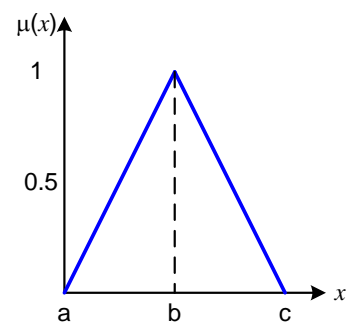

(a)

Fig. 3 (a) Triangular MF, (b) Trapezoidal MF

When Trapezoidal MF is used as a mapping function, it will work to suppress all pixels that can cause increased dynamic range within its texture classes, depending on the function parameter used. The Trapezoidal MF is mathematically expressed by

$f(x, a, b, c, d)=\left\{\begin{array}{cc}0 & x \leq a \\ \frac{x-a}{b-a} & a \leq x \leq b \\ 1 & b \leq x \leq c \\ \frac{d-x}{d-c} & c \leq x \leq d \\ 0 & d \leq x\end{array}\right.$

If the image data type is double / float then the gray-level dynamic is in range $\{0 \ldots 1\}$. If the Trapezoidal MF used has parameters $a=b=0, c=0.5$, and $d=1$ then all the pixels intensity values that meet $I(x, y) \leq 0.5$ will be mapped into $g(x, y)=1$, whereas the pixel intensity value that satisfies $I(x, y)>0.5$ will be mapped into $g(x, y)=(1-I(x, y)) /$ 0.5 .

This technique is applied to every component $\mathrm{R}, \mathrm{G}$ and $\mathrm{B}$ of the RGB image. The $c$ parameter of Trapezoidal MF is determined by the proportion of the number of pixel intensity values of each component to the total pixel intensity value of the RGB image with size $M \times N$, expressed by the following formula

$$
\operatorname{sumR}=\sum_{i=1}^{M} \sum_{j=1}^{N} I_{R}(i, j) \quad \operatorname{sum} B=\sum_{i=1}^{M} \sum_{j=1}^{N} I_{B}(i, j)
$$

$\operatorname{sum} G=\sum_{i=1}^{M} \sum_{j=1}^{N} I_{G}(i, j)$

$r=\frac{\operatorname{sum} R}{\operatorname{sum} R+\operatorname{sum} G+\operatorname{sum} B}$

$g=\frac{\text { sum } G}{\operatorname{sum} R+\operatorname{sum} G+\operatorname{sum} B}$

$b=\frac{\operatorname{sum} B}{\operatorname{sum} R+\operatorname{sum} G+\operatorname{sum} B}$

Eq. (6) is used to generate 3 (three) gray images as the result of gray level mapping by using Trapezoidal MF, which expressed by

$$
\begin{aligned}
& R_{f u z z}(x, y)=f(I(x, y, 1), 0,0, r, 1) \\
& G_{f u z z}(x, y)=f(I(x, y, 2), 0,0, g, 1) \\
& B_{f u z z}(x, y)=f(I(x, y, 3), 0,0, b, 1)
\end{aligned}
$$

The mapping results of the three components of the image are then combined into a fuzzy-based image by using the following formula

$$
\begin{aligned}
& I_{f u z z}(x, y, 1)=R_{f u z z}(x, y) \\
& I_{f u z z}(x, y, 2)=G_{f u z z}(x, y) \\
& I_{f u z z}(x, y, 3)=B_{f u z z}(x, y)
\end{aligned}
$$

The operation is shown in Fig. 4.

\section{FEATURE EXTRACTION}

The image features are expressed by a feature descriptor in the form of labels to ensure low dimensional representation. In this study, feature labels are generated from two-dimensional image.

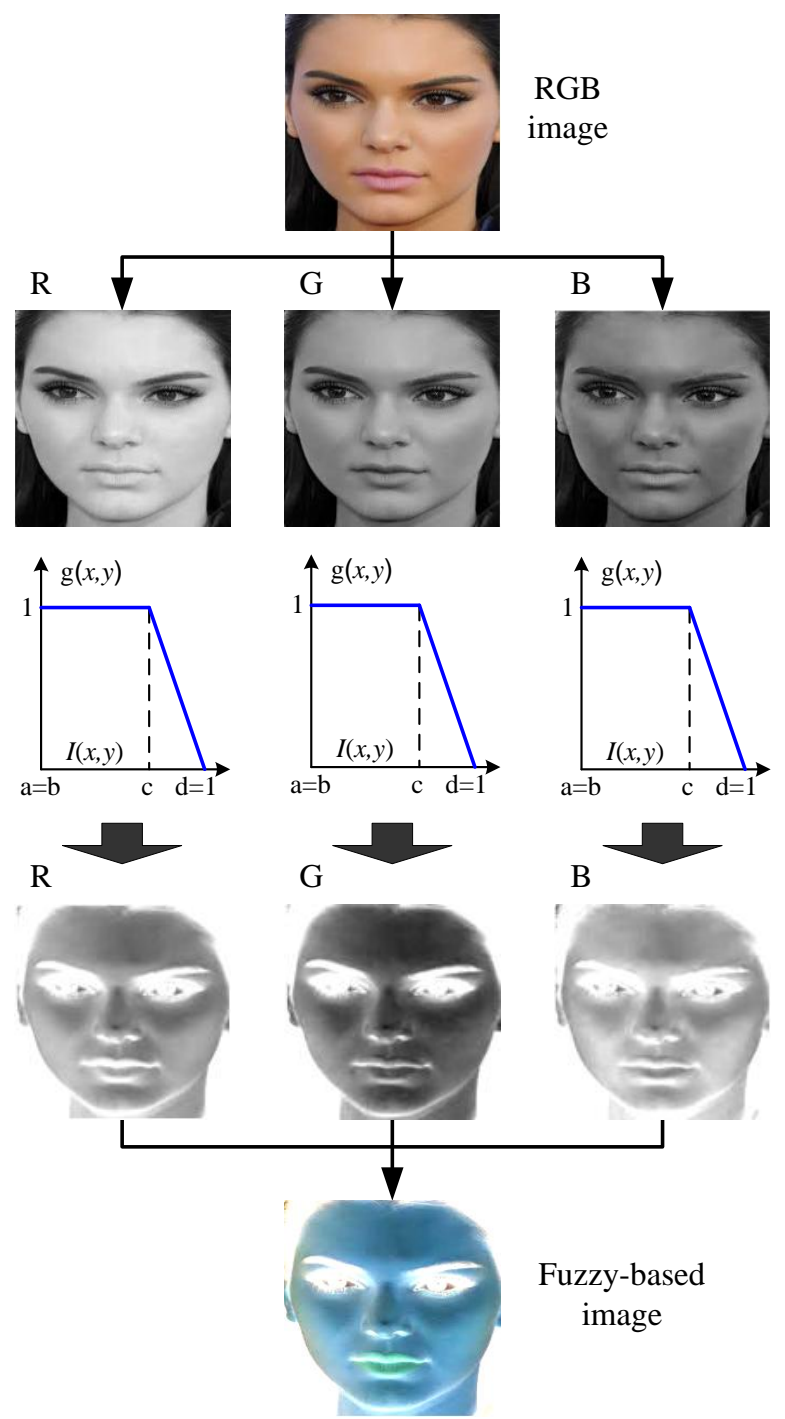

Fig. 4 Fuzzy-based image generation

Therefore the RGB image needs to be converted into a twodimensional image by applying the AND fuzzy operator between $\mathrm{R}, \mathrm{G}$, and $\mathrm{B}$ components expressed by the following formula:

$I_{\text {feat }}(x, y)=\left(\begin{array}{l}I_{f u z z}(x, y, 1), \\ \min \left(I_{f u z z}(x, y, 2), I_{f u z z}(x, y, 3)\right)\end{array}\right)$ 
The True Fuzzy-based image and the LWP texture image are visualized as negative images so they need to be converted into the true image before extracting their features, which expressed by

$I_{\text {fuzz_true }}(x, y)=1-I_{\text {fuzz }}(x, y)$
$I_{\text {LWP_true }}(x, y)=1-I_{L W P}(x, y)$

The use of AND fuzzy operator is illustrated in Fig. 5.

In order for the feature label to have a specified length, the image features need to be converted into a new image feature that has a length of column $=$ the length of the feature label. If the length of the feature label is $L$, then

$$
\begin{aligned}
& I_{\text {feat_mean }}(j)=\sum_{i=1}^{M} I_{\text {feat }}(i, j) \quad j=1 \ldots N \\
& I_{\text {newfeat }}=\text { reshape }\left(I_{\text {feat_mean }},\left[\begin{array}{ll}
N / L & L
\end{array}\right]\right)
\end{aligned}
$$

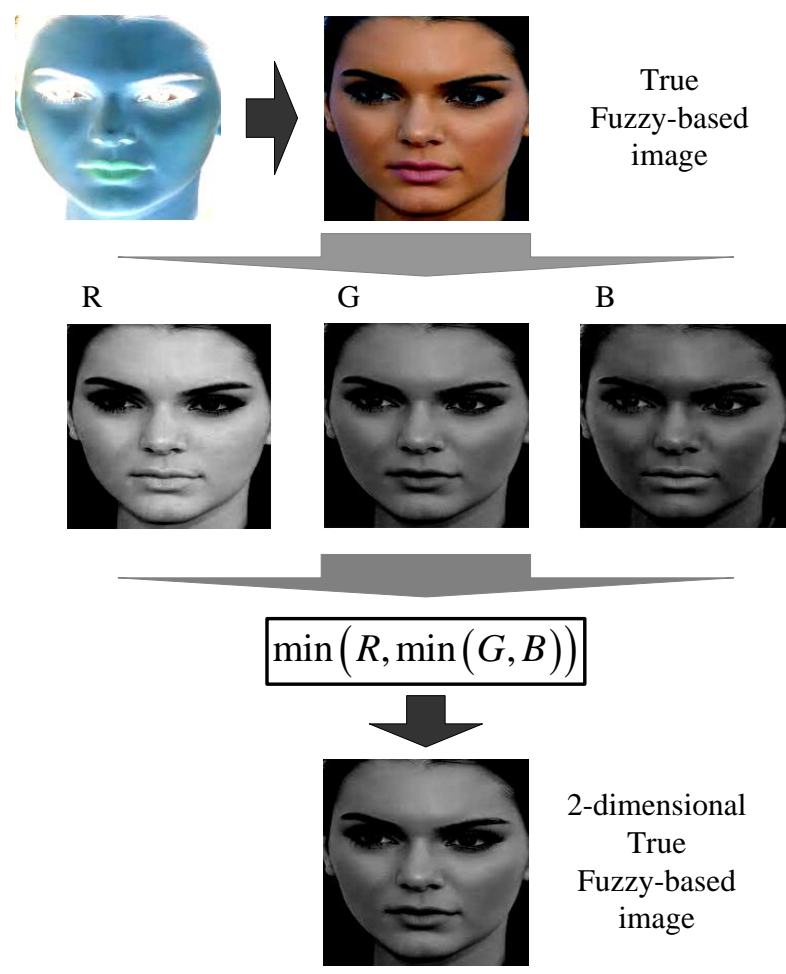

Fig. 5 The use of AND fuzzy operator

The feature label is expressed by

$$
F(j)=\sum_{i=1}^{N / L} I_{\text {newfeat }}(i, j) \quad j=1 \ldots L
$$

\section{PERFORMANCE MEASUREMENTS}

\subsection{Peak to Average Power Ratio (PAPR)}

The PAPR of a signal is defined as the ratio of the maximum instantaneous power to its average power. High PAPR results indicate that the signal has a large dynamic range [19], and leads to signal distortion and power inefficiency [20]. When expressed in decibels then vice versa, and expressed by

$\operatorname{PAPR}(x(t))=10 * \log _{10}\left(\frac{\max \left(|x(t)|^{2}\right)}{\frac{1}{N L} \sum_{i=1}^{N L}|x(i)|^{2}}\right) d B$
If the feature label is considered a signal then $x(t)=F$, and $N L=N / L$. In this case, a high PAPR indicates that the feature label has a small dynamic range in the texture classes, so the probability of having multiple features is very small. In other words the resulting feature label is unique.

\subsection{MAPE (Mean Absolute Percentage Error) and MSE} (Mean Square Error)

In this study, the performance measurements of uniqueness level changes caused by variations of illumination effects on the image is done by using MAPE (Mean Absolute Percentage Error) expressed by

$$
\begin{aligned}
& A P E(i)=\left(\frac{P A P R \text { change }}{P A P R}\right) \times 100 \% \\
& M A P E=\frac{1}{N} \sum_{i=1}^{N} A P E(i)
\end{aligned}
$$

Where $P A P R$ changes is the difference between the $P A P R$ of the original feature label and the $P A P R$ of the feature label affected by the illumination variation, $N$ is the number of the test image affected by the illumination variation.

Performance measurement of robustness of feature labels caused by variations of illumination effects using the $M S E$ expressed by

$M S E=\frac{1}{N L} \sum_{i=1}^{N L}\left(F_{\text {ori }}(i)-F_{\text {affected }}(i)\right)^{2}$

\section{RESULTS AND ANALYSIS}

In this study, as a test image is an RGB image that has a size of $253 \times 199 \times 3$. The image needs to be converted into a template that has the same row-column size to get uniform information from the test image. This study used the 200x200 template so that the test image as shown in Fig. 6. By applying Eq. (1), (2) and (3) on each component of the test image then obtained the LWP texture image $\left(I_{L W P}\right)$ as shown in Fig. 7. By applying Eq. (5), (6), (7) and (8) on the test image then obtained the Fuzzy-based image $\left(I_{f u z z}\right)$ as shown in Fig. 8.

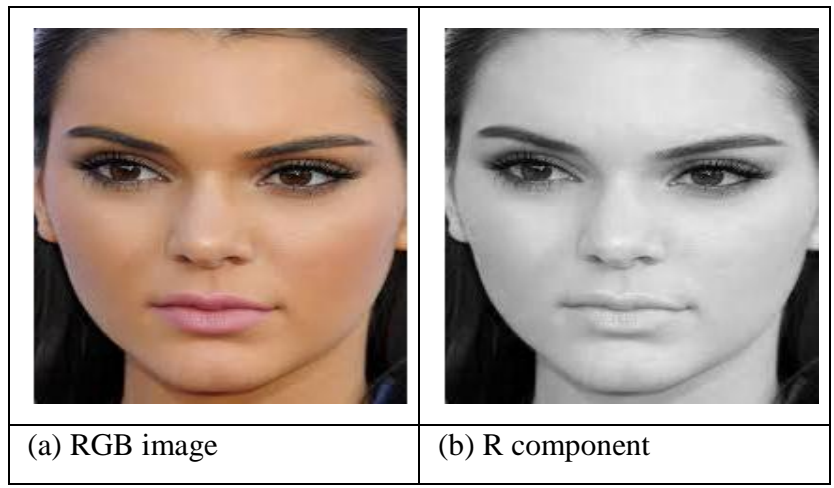




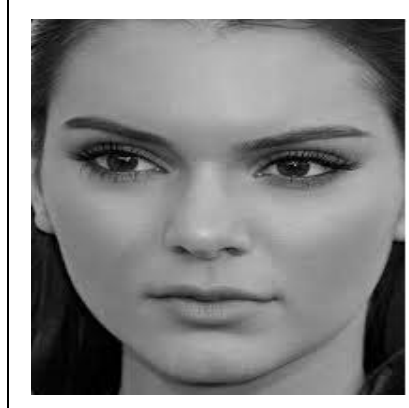

(c) G component (d) B component

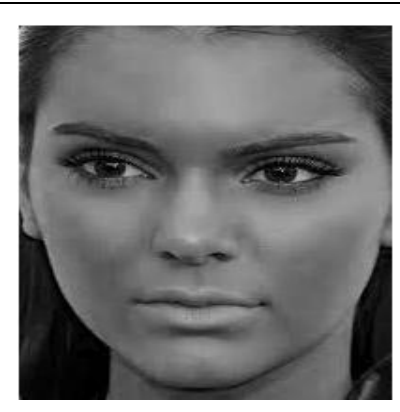

Fig. 6 Test image after convert into a template of $200 \times 200 \times 3$ size

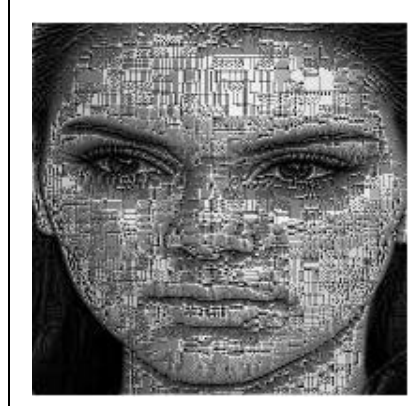

(a) $\mathrm{R}$ component

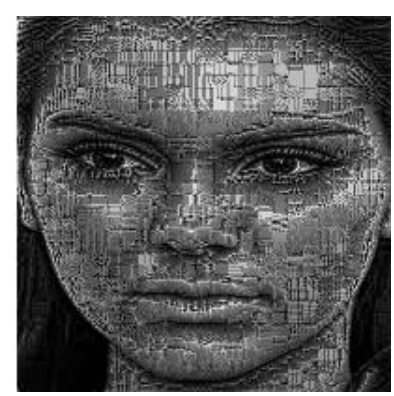

(c) B component

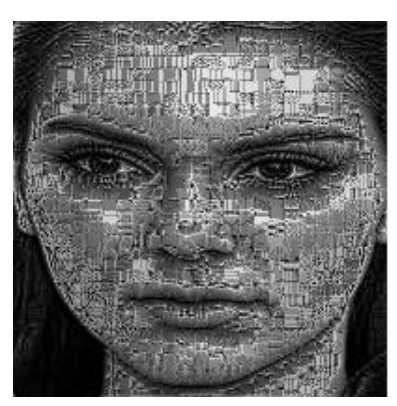

(b) G component

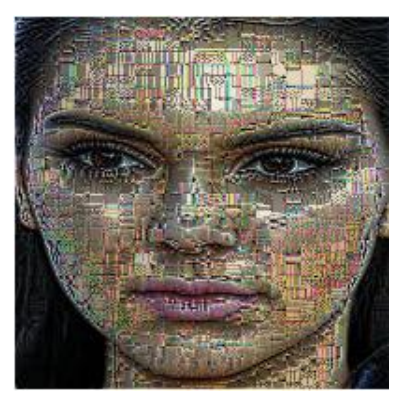

(d) LWP texture
Fig. 7 LWP texture image

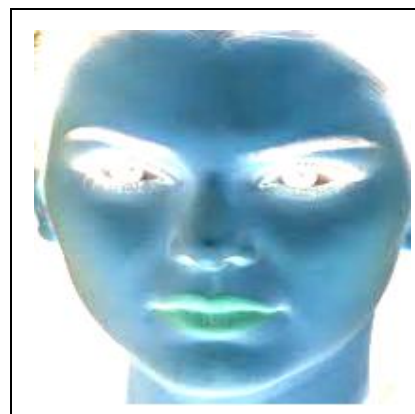

(a) Fuzzy-based image

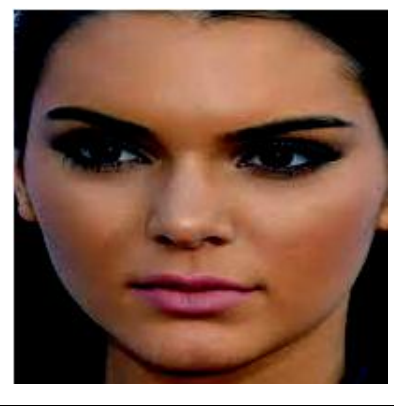

(b) True fuzzy-based image
Fig. 8 Fuzzy-based image

By applying Eq. (5), (6), (7) and (8) on the LWP texture image then obtained the Fuzzy-based LWP texture image as shown in Fig. 9.

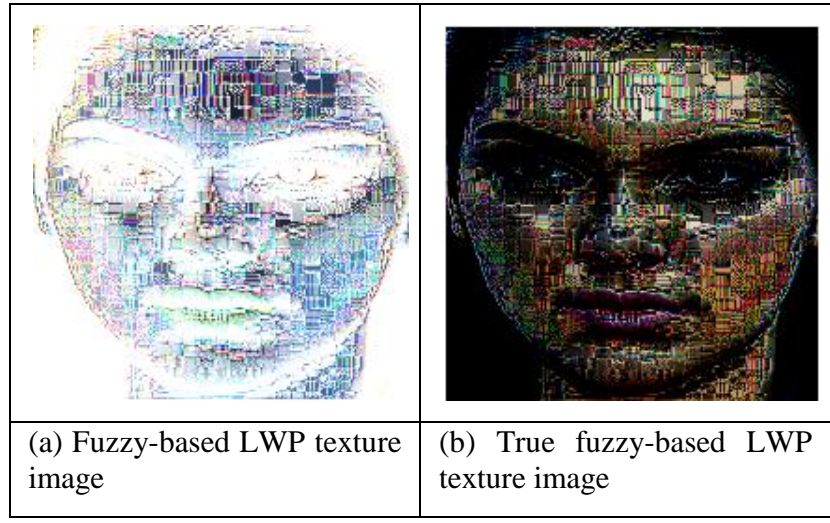

Fig. 9 Fuzzy-based LWP texture image

By applying Eq. (9), (10), (11) and (12) on the images as shown in Fig. 6 (a), 7 (d), 8 (b), and 9 (b) then obtained the feature labels as shown in Table 1 and Fig. 10.

Table 1 Feature labels

\begin{tabular}{|c|c|c|c|c|}
\hline Label & $I_{\text {ori }}$ & $I_{L W P}$ & $I_{f u z z}$ & $I_{f u z z L W P}$ \\
\hline 1 & 0.2048 & 0.1327 & 0.0537 & 0.0192 \\
\hline 2 & 0.2780 & 0.1817 & 0.1211 & 0.0378 \\
\hline 3 & 0.3391 & 0.2252 & 0.1631 & 0.0453 \\
\hline 4 & 0.3722 & 0.2507 & 0.1909 & 0.0553 \\
\hline 5 & 0.4132 & 0.2880 & 0.2268 & 0.0807 \\
\hline 6 & 0.5015 & 0.3598 & 0.2875 & 0.1169 \\
\hline 7 & 0.5213 & 0.3741 & 0.3089 & 0.1250 \\
\hline 8 & 0.5230 & 0.3683 & 0.3076 & 0.1232 \\
\hline 9 & 0.5573 & 0.3796 & 0.3581 & 0.1306 \\
\hline 10 & 0.5952 & 0.4409 & 0.4134 & 0.2054 \\
\hline 11 & 0.5860 & 0.4414 & 0.3984 & 0.2098 \\
\hline 12 & 0.5864 & 0.4384 & 0.3969 & 0.2082 \\
\hline 13 & 0.5760 & 0.4080 & 0.3896 & 0.1714 \\
\hline 14 & 0.5563 & 0.4221 & 0.3698 & 0.1979 \\
\hline 15 & 0.5471 & 0.4282 & 0.3522 & 0.2055 \\
\hline 16 & 0.5454 & 0.4283 & 0.3448 & 0.2027 \\
\hline 17 & 0.5410 & 0.3959 & 0.3446 & 0.1630 \\
\hline 18 & 0.5094 & 0.3697 & 0.3157 & 0.1388 \\
\hline 19 & 0.3682 & 0.2593 & 0.2168 & 0.0929 \\
\hline 20 & 0.2515 & 0.1797 & 0.1152 & 0.0460 \\
\hline
\end{tabular}




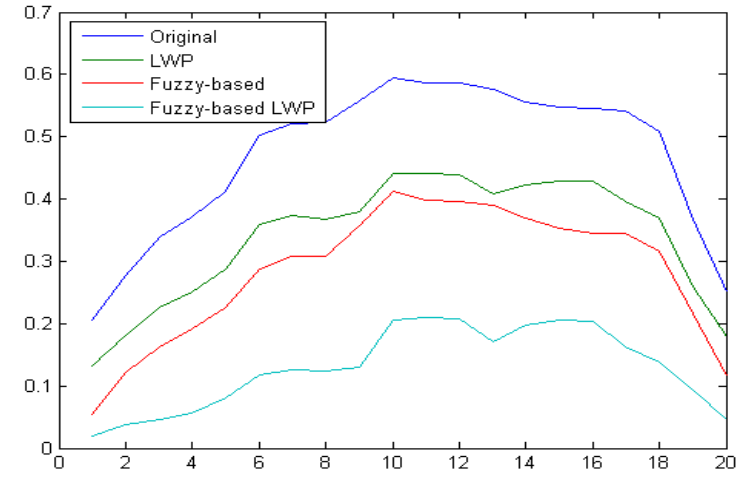

Fig. 10 Feature labels visualization

Performance of the feature labels as shown in Table 1 are measured by PAPR with the results as shown in Table 2 . Referring to Table 2, the feature labels of Fuzzy-based LWP have the highest PAPR of $10.0702 \mathrm{~dB}$. This indicates that the feature labels of Fuzzy-based LWP have a higher level of uniqueness than others.

Table 2 PAPR measurement results

\begin{tabular}{|l|c|c|c|c|}
\hline Perf. & $I_{\text {ori }}$ & $I_{L W P}$ & $I_{\text {fuzz }}$ & $I_{\text {fuzzLWP }}$ \\
\hline PAPR (dB) & 4.0534 & 5.5109 & 6.5486 & 10.0702 \\
\hline
\end{tabular}

To test the robustness of feature labels produced from the proposed method against the illumination effects, it also tries to be applied to the other test images as shown in Fig. 11. The other type of test images is the original image that has been affected by the illumination effect so it looks less $(-25 \%)$ or brighter $(+25 \%)$ than the original.

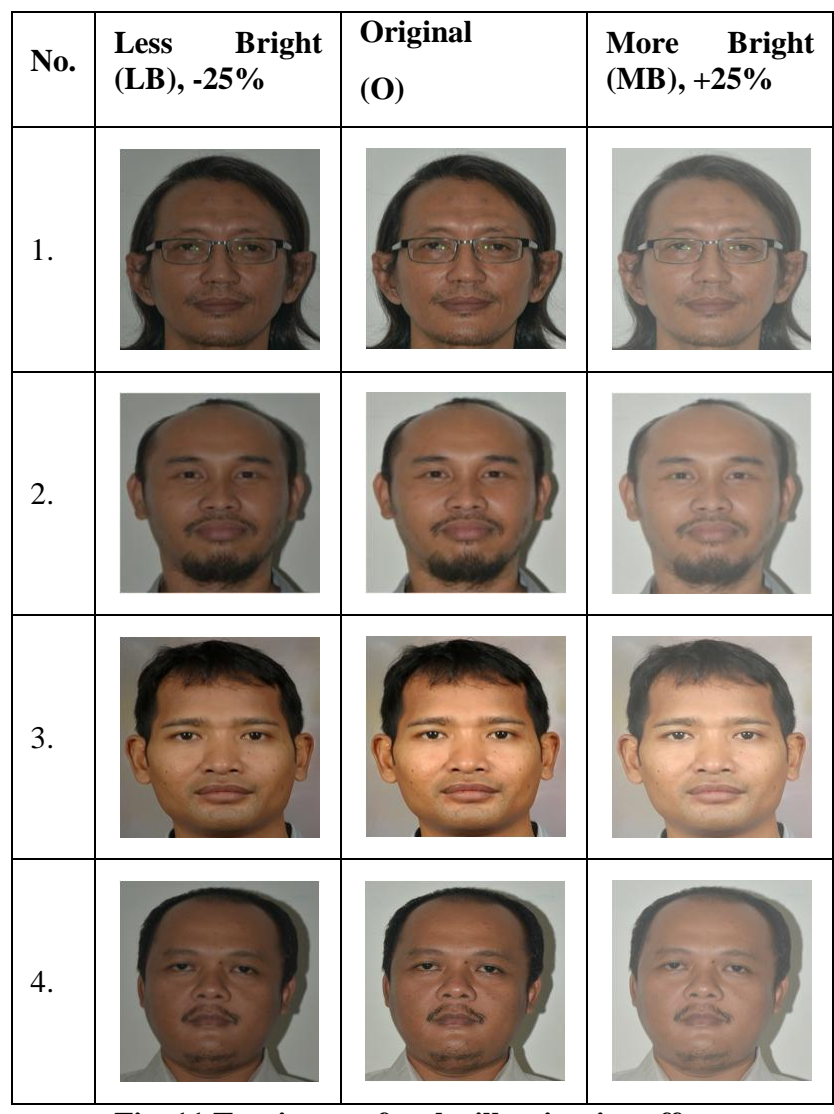

Fig. 11 Test images for the illumination effects measurement
Performance measurement is done in two ways, as follows:

1. PAPR measurement is performed on each type of test image $(\mathrm{O}, \mathrm{MB}$, and $\mathrm{LB})$. APE (Absolute Percentage Error) measurement is performed on MB and LB toward O. MAPE measurements (Mean APE) are the average of both measurements. The results are shown in Table 3 .

2. The measurement of MSE (Mean Square Error) is performed on the feature label value of $\mathrm{MB}$ and $\mathrm{LB}$ toward the feature label value of O. MMSE (Mean MSE) is the average of both measurements. The results are shown in Table 4.

Referring to Table 3, the feature labels of Fuzzy-based LWP have a smaller average MAPE of PAPR measurement of all the test images, which changes the uniqueness of the label feature due to the illumination effect on the image by $8.34 \%$. Referring to Table 4, the feature labels of Fuzzy-based LWP have a smaller average MMSE measurement of all the test images, which changes the robustness of the label feature due to the illumination effect on the image by 0.0031 .

Table 3 MAPE of PAPR measurement of all test images

\begin{tabular}{|c|c|c|c|c|c|}
\hline No & Type & $I_{\text {ori }}$ & $I_{L W P}$ & $I_{f u z z}$ & $I_{f u z z L W P}$ \\
\hline \multirow{6}{*}{1} & (O) & 6.388 & 8.691 & 11.697 & 15.118 \\
\hline & (MB) & 4.130 & 6.748 & 7.953 & 12.686 \\
\hline & (LB) & 7.687 & 8.672 & 15.910 & 14.637 \\
\hline & $\mathrm{APE}_{\mathrm{O} \_\mathrm{MB}}$ & $35.34 \%$ & $22.36 \%$ & $32.01 \%$ & $16.08 \%$ \\
\hline & $\mathrm{APE}_{\mathrm{O} \_\mathrm{LB}}$ & $20.33 \%$ & $0.21 \%$ & $36.02 \%$ & $3.18 \%$ \\
\hline & MAPE 1 & $27.84 \%$ & $11.28 \%$ & $34.01 \%$ & $9.63 \%$ \\
\hline \multirow{6}{*}{2} & (O) & 5.131 & 6.888 & 9.309 & 11.662 \\
\hline & $(\mathrm{MB})$ & 3.449 & 5.634 & 6.226 & 10.050 \\
\hline & (LB) & 6.417 & 6.747 & 13.322 & 11.209 \\
\hline & $\mathrm{APE}_{\mathrm{O} \_\mathrm{MB}}$ & $32.78 \%$ & $18.21 \%$ & $33.12 \%$ & $13.83 \%$ \\
\hline & $\mathrm{APE}_{\mathrm{O} \_\mathrm{LB}}$ & $25.07 \%$ & $2.04 \%$ & $43.10 \%$ & $3.89 \%$ \\
\hline & MAPE 2 & $28.93 \%$ & $10.13 \%$ & $38.11 \%$ & $8.86 \%$ \\
\hline \multirow{6}{*}{3} & (O) & 4.743 & 6.834 & 8.424 & 13.471 \\
\hline & (MB) & 3.130 & 5.590 & 5.719 & 11.739 \\
\hline & (LB) & 6.036 & 6.779 & 13.205 & 13.228 \\
\hline & $\mathrm{APE}_{\mathrm{O} \_\mathrm{MB}}$ & $34.01 \%$ & $18.19 \%$ & $32.12 \%$ & $12.85 \%$ \\
\hline & $\mathrm{APE}_{\mathrm{O} \_ \text {LB }}$ & $27.26 \%$ & $0.81 \%$ & $56.75 \%$ & $1.80 \%$ \\
\hline & MAPE 3 & $30.63 \%$ & $9.50 \%$ & $44.43 \%$ & $7.33 \%$ \\
\hline \multirow{6}{*}{4} & (O) & 5.421 & 7.150 & 9.707 & 12.117 \\
\hline & (MB) & 3.625 & 5.775 & 6.586 & 10.847 \\
\hline & (LB) & 6.705 & 7.012 & 13.721 & 11.557 \\
\hline & $\mathrm{APE}_{\mathrm{O} \_\mathrm{MB}}$ & $33.13 \%$ & $19.22 \%$ & $32.15 \%$ & $10.48 \%$ \\
\hline & $\mathrm{APE}_{\mathrm{O} \_ \text {LB }}$ & $23.67 \%$ & $1.92 \%$ & $41.35 \%$ & $4.62 \%$ \\
\hline & MAPE 4 & $28.40 \%$ & $10.57 \%$ & $36.75 \%$ & $7.55 \%$ \\
\hline \multicolumn{2}{|c|}{ Average MAPE } & $28.95 \%$ & $10.37 \%$ & $38.33 \%$ & $8.34 \%$ \\
\hline
\end{tabular}


Table 4 MSE measurement of all the test images

\begin{tabular}{|c|c|c|c|c|c|}
\hline No. & MSE & $I_{\text {ori }}$ & $I_{L W P}$ & $I_{f u z z}$ & $I_{f u z z L W P}$ \\
\hline \multirow{3}{*}{1} & $(\mathrm{O}-\mathrm{MB})$ & 0.0246 & 0.0081 & 0.0269 & 0.0026 \\
\hline & $(\mathrm{O}-\mathrm{LB})$ & 0.0630 & 0.0073 & 0.0636 & 0.0021 \\
\hline & MMSE 1 & 0.0438 & 0.0077 & 0.0452 & 0.0023 \\
\hline \multirow{3}{*}{2} & $(\mathrm{O}-\mathrm{MB})$ & 0.0192 & 0.0060 & 0.0320 & 0.0040 \\
\hline & $(\mathrm{O}-\mathrm{LB})$ & 0.0630 & 0.0045 & 0.0939 & 0.0027 \\
\hline & MMSE 2 & 0.0411 & 0.0052 & 0.0629 & 0.0033 \\
\hline \multirow{3}{*}{3} & $(\mathrm{O}-\mathrm{MB})$ & 0.0187 & 0.0050 & 0.0223 & 0.0033 \\
\hline & $(\mathrm{O}-\mathrm{LB})$ & 0.0630 & 0.0045 & 0.0988 & 0.0027 \\
\hline & MMSE 3 & 0.0409 & 0.0047 & 0.0605 & 0.0030 \\
\hline \multirow{3}{*}{4} & $(\mathrm{O}-\mathrm{MB})$ & 0.0208 & 0.0069 & 0.0318 & 0.0046 \\
\hline & $(\mathrm{O}-\mathrm{LB})$ & 0.0630 & 0.0052 & 0.0865 & 0.0029 \\
\hline & MMSE 4 & 0.0419 & 0.0061 & 0.0591 & 0.0037 \\
\hline \multicolumn{2}{|c|}{ Average MMSE } & 0.0419 & 0.0059 & 0.0570 & 0.0031 \\
\hline
\end{tabular}

This can be explained as follows. The feature labels of the Fuzzy-based image changes drastically when exposed to variations of illumination effects. This is because the Fuzzybased image is based on the proportion of the weight of each component $\mathrm{R}, \mathrm{G}$, and $\mathrm{B}$ on the overall pixel intensity value. The feature labels of LWP texture image change quite small when exposed to variations of illumination effects. This is because LWP texture image perform local weighting on each pixel based on the amount of binary code built from the threshold value between neighboring pixels and their center of pixel. This means that changes in pixel intensity values caused by variations in illumination effects will be minimized. The ability of a Fuzzy-based image concept that filters out the pixel intensity values that can lead to increased dynamic range of texture classes further enhances the feature labels of LWP texture image when combined.

\section{CONCLUSION}

In this paper, LWP has been used to create the LWP texture image from RGB image. By applying the concept of Fuzzybased image generation on LWP texture image then obtained Fuzzy-based LWP texture image. This texture image is then used to generate the feature descriptor in the form of labels. From the experimental results, it was found that the feature labels of Fuzzy-based LWP have higher PAPR than others. In this case, used as a comparison is the feature label generated from the original image, LWP texture image, and Fuzzy-based image. It can be concluded that the feature label of the Fuzzybased LWP texture image has a higher level of uniqueness than the others. From the results of the illumination effects test $(+/-25 \%)$ against the original image, the feature labels of Fuzzy-based LWP only slight changes in uniqueness and robustness.

Future work is how to improve the performance of Fuzzybased LWP feature labels in terms of their uniqueness, robust against larger illumination variations, and much lower dimensional representations.

\section{ACKNOWLEDGMENTS}

The Authors would like to express their heartfelt thanks to The Modern Computing Research Center, Department of Information Technology, State Polytechnic of Samarinda for giving all their support.

\section{REFERENCES}

[1] S. Fekri-Ershad, "Innovative Texture Database Collecting Approach and Feature Extraction Method based on Combination of Gray Tone Difference Matrixes, Local Binary Patterns, and K-means Clustering," presented at the First National Conference on Computer, Information Technology and Communication (CCITC 2014), Marvdasht, Fars, Iran, 2014.

[2] S. L. Li Liu, Paul W. Fieguth, Yulan Guo, Xiaogang Wang, and Matti Pietikäinen, "Median Robust Extended Local Binary Pattern for Texture Classification," IEEE TRANSACTIONS ON IMAGE PROCESSING 2016.

[3] E. H. K. Mandeep Kaur, "An Empirical Study on Texture Feature Extraction Methods for Image Retriveal," International Journal of Advanced Research in Computer Science, vol. 8, p. 4, May, 20172017.

[4] N. M. W. Mayuri R. Wankhade, "Feature Extraction of Edge Detected Images," International Journal of Computer Science and Mobile Computing (IJCMSC), vol. 6, pp. 336 -345, June, 20172017.

[5] F. L. M. Mostafa G. Saeed, Zaid Ahmed Aljawaryy, "Content-Based Image Retrieval by Multi Features Extraction and K-Means Clustering," International Journal of Electrical, Electronics and Computers (EEC Journal) vol. 2, p. 11, May-June, 20172017.

[6] A. S. Noor Saffazura, Marina Ismail, Nursuriati Jamil, "Feature Extraction Technique for Human Gait Video Analysis," Journal of Engineering and Applied Sciences, Medwell Journals, vol. 12, pp. 534-541, 2017.

[7] V. K. Rajkumar Goel, Saurabh Srivastava, A. K. Sinha, "A Review of Feature Extraction Techniques for Image Analysis," International Journal of Advanced Research in Computer and Communication Engineering, vol. 6, February, 20172017.

[8] S. T. Suvarnsing G. Bhable, Hanumant Gite, Siddharth Dabhade, K. V. Kale, "Review on Face, Ear and Signature for Human Identification," International Journal of Computer Applications (IJCA), vol. 180, 2018.

[9] X. Z. Ting Chen, Liang Dai, Licheng Zhang, and Jiarui Wang, "A Novel Texture Feature Description Method Based on the Generalized Gabor Direction Pattern and Weighted Discrepancy Measurement Model," Symmetry, vol. 8, 2016.

[10] D. S. R. G. Vaishnavi S. Mehekare, "Detection of Brain Tumor Using Discrete Wavelet Transform, PCA \& KSVM " International Journal of Innovative Research in Computer and Communication Engineering (IJIRCCE), vol. 5, pp. 10228-10236, May, 20172017.

[11] C. N. D. Pallavi B. Patinge, "Local Binary Pattern Base Face Recognition System," International Journal of Science, Engineering and Technology Research (IJSETR), vol. 4, May, 20152015. 
[12] D. D. J. P. Pranita R. Chavan, "Face Recognition using Local Derivative Pattern Face Descriptor," International Journal Of Engineering And Computer Science (IJECS), vol. 3, pp. 8830-8834, October, 2014 2014.

[13] G. K. M. P. Venkata Satya Kumar Gangavarapu, "Local Tri-directional Weber Patterns: A New Descriptor for Texture and Face Image Retrieval," International Journal of Computer Science and Information Technologies (IJCSIT), vol. 7, pp. 1571-1577, 2016.

[14] L. S. A. Siddiqui Almas, TelgadRupali L., Deshmukh P. D., "Feature Level Fusion for Fingerprint using Neural Network for Person Identification," in IJCA Proceedings on International Conference on Cognitive Knowledge Engineering India, 2016, pp. 41-45.

[15] T. M. Steren Chabert, Rodrigo Riveros, Maximiliano Godoy, Alejandro Veloz, and P. C. Rodrigo Salas, "Applying Machine Learning and Image Feature Extraction Techniques to The Problem of Cerebral Aneurysm Rupture," Research Ideas and Outcomes, vol. 3, 10 January, 20172017.

[16] S. G. Manisha Nirgude, "Iris Recognition System based on Multi-resolution Analysis and Support Vector
Machine," International Journal of Computer Applications (IJCA), vol. 173, 2017.

[17] R. H. J. Abbas H. Hassin Alasadi, "Fingerprint Verification System based on Active Forgery Techniques," International Journal of Computer Applications (IJCA), vol. 180, pp. 6-10, January, 2018 2018.

[18] R. H. H. Khalil Ibrahim Alsaif, "Study the Effect of Threshold Value on Object Detection," International Journal of Computer Applications (IJCA), vol. 179, pp. $10-13,2018$.

[19] K. N. M. Manjula A. V., "PAPR Reduction in OFDM Systems using RCF and SLM Techniques," International Journal of Computer Applications (IJCA), vol. 158, pp. 6-9, January, 20172017.

[20] S. N. J. Dike U. Ike, Charles Ndujiuba, "Modified Selected Mapping Technique for Peak-to-Average Power Ratio Reduction in Orthogonal Frequency Division Multiplexing Systems," International Journal of Current Engineering and Technology (IJCET), vol. 7, pp. 1427-1432, August, 20172017. 